\title{
Urgences
}

\section{L'ange de la solitude ou l'a-modernité comme une geste lyrique}

\section{Frances Fortier}

Numéro 28, mai 1990

Le roman comme poétique

URI : https://id.erudit.org/iderudit/025591ar

DOI : https://doi.org/10.7202/025591ar

Aller au sommaire du numéro

Éditeur(s)

Urgences

ISSN

0226-9554 (imprimé)

1927-3924 (numérique)

Découvrir la revue

Citer cet article

Fortier, F. (1990). L'ange de la solitude ou l'a-modernité comme une geste

lyrique. Urgences, (28), 79-93. https://doi.org/10.7202/025591ar d'utilisation que vous pouvez consulter en ligne.

https://apropos.erudit.org/fr/usagers/politique-dutilisation/ 


\title{
L'ange de la solitude ou l'a-modernité comme une geste lyrique
}

\author{
Frances Fortier
}

Cette sensibilité très voilée à l'égard, non de la beauté formelle, définitive, mais de l'indication fulgurante d'une manifestation sans autre nom que la poésie...

Jean Genet

Il est de bon ton de lire Marie-Claire Blais dans le champ très exactement balisé de l'écriture au féminin, champ qui recoupe - et sature? - celui de la modernité textuelle. Sa prose poétique multiplie les pistes de questionnement: de lectures psychanalytiques en investigations formelles, l'œuvre se voit constamment jaugée à l'aune du vocabulaire critique dominant. Peut-on, sans encourir l'anathème, s'autoriser des seules clés que propose L'ange de la solitude ${ }^{1}$, le dernier roman de Blais, pour en montrer l'appartenance à une esthétique "démodée"? Une telle lecture, sacrilège il va sans dire, s'ingénierait pourtant à réconcilier l'écart manifeste entre le discursif et le narratif, à récupérer la pratique intertextuelle pour l'inscrire dans le procès dynamique de l'œuvre, bref tenterait la mise en correspondance de tous les signes du texte. Bien que le mot ne soit plus à la mode, comme les "communes" d'ailleurs, il me semble que la poésie "symboliste " est au cœur du projet d'écriture. L'affirmation d'une telle esthétique, si elle se lisait dans le texte, n'irait cependant pas sans l'inscription factuelle du poétique. Hypothèse(s)...

\section{L'enjeu romanesque}

Le canevas diégétique de L'ange de la solitude est minimal. Six personnages féminins, aux noms énigmatiques, schématisés par leurs passions individuelles, vivent en commune. Doudouline la musicienne, L'Abeille la peintre,

Marie-Claire Blais, L'ange de la solitude (ici AS), Montréal, VLB éditeur, 1989. 
Johnie l'écrivaine, Gérard la toxicomane, Thérèse la travailleuse sociale et Polydor la mystique partagent une même marginalité: leur jeunesse sans avenir, leurs amours homosexuelles s'engluent dans les relents d'ivresse et une pesante oisiveté. L'une d'entre elles, Gérard, mourra avant d'avoir vingt ans. Des espaces clos, la maison, le Club, les fonds de cour, espaces fermés d'où l'on s'échappe vers des contrées lointaines et mystérieuses, des continents interdits. Un temps circulaire, ponctué par les saisons et le rythme lunaire qui gèrent la lumière autant que les états d'âmes. L'univers lesbien de L'ange de la solitude assume tous ces postulats, et les multiplie: Sophie, l'image de la mère, Paula, l'initiatrice sexuelle dépravée, et la mâlitude, résolument confinée à la figuration, 's'inscrivent comme autant de fragments juxtaposés, sans incidence réelle sur la trame romanesque. L'ensemble forme une constellation statique, où viennent se greffer en contrepoint les constantes de l'univers blaisien: l'enfant rimbaldien, le caractère sacré de l'écriture et de l'art, le monde apocalyptique. Tout est figé, immobile, léthargique, assommé d'angoisse, sans relief. L'écriture, pour une part, redouble cet immobilisme ${ }^{2}$. Ici aussi, on retrouve la manière blaisienne, à tout le moins celle qui est mise de l'avant dans ses derniers textes: narration entièrement à l'imparfait, monologues intérieurs non assumés par un $\mathrm{JE}$, absence de dialogues, focalisations multiples des personnages qui s'épient et se jugent mutuellement, auto-engendrement de l'écriture par réembrayage textuel ou métonymique. Ces procédés romanesques, apparentés à la modernité littéraire et en l'occurrence parfaitement maîtrisés, concourent à l'échafaudage d'un jeu complexe de miroirs, où cependant rien n'est à voir que le «cristal » transparent de l'ange de la solitude.

Et si l'enjeu était ailleurs? Ailleurs que dans ce mimétisme de l'écriture, qui traduit dans son épuration formelle le noyau même de la solitude? Notre hypothèse postule une tension entretenue, et nullement résolue, entre un discours idéologiquement marqué par l'Histoire - et assumé par le personnage de l'écrivaine Johnie - et une pratique intertextuelle qui vient polariser et dynamiser, d'un autre lieu, à la fois le récit et son écriture.

2 A cet égard, Louise Milot a bien montré, dans a Le dernier Marie-Claire Blais ", Lettres québécoises, $n^{\circ} 55$, automne 1989, p. 24-25, ce que j'appelle la faillite du narratif. 


\section{"L'Univers de Johnie" ou la lecture de signes analo- giques diffractés}

L'inscription du personnage de Johnie dans le titre de la première partie du texte a une fonction de désignation. L'élection de l'écrivaine comme pôle central de l'univers décrit pose la figure de l'écriture au sommet de la hiérarchie des disciplines artistiques convoquées par le texte. Bien que rien ne la distingue dans les modalités d'énonciation - puisque jamais un JE ne s'accapare le discours -, Johnie, passionnée d'écriture, boursière d'université, rédige un essai intitulé «De Sapho à Radclyffe Hall» 3 . Sous cette figure se trouvent ainsi indexées la lecture et la réécriture d'un discours multiple, historique et culturel, discours d'hier et d'aujourd'hui, discours du privé et du social: « on aurait pu croire que Johnie était victime de cette passion du mot écrit, fureur sacrée dont Gérard était jalouse " $(A S, 14)$.

Univers du langage donc, univers du sacré, mais aussi univers du sensible. Les amours malheureuses, les disparités sociales, les racismes de tous ordres, la violence du monde contemporain apparaissent dans le tissu discursif sous la figure thématisée de la solitude. Une solitude pensée comme un rejet, comme un défaut d'harmonie, ainsi que le précise la citation en exergue:

L'ange de la solitude, c'est-à-dire un être de plus en plus inhumain, cristallin, autour de qui se dévéloppent les bandes d'une musique basée sur le contraire de l'harmonie, ou plutôt une musique qui est ce qui demeure quand l'harmonie est usée.

La grande quête de l'harmonie menée par les filles de la commune qui toutes poursuivent *le rêve de changer le monde", qui est l'objet-valeur du programme narratif, devient la clé de voûte de toute l'organisation discursive. L'harmonie visée, c'est d'abord le rêve de la fusion individuelle: l'amour, premier facteur d'harmonie, devrait unir les couples de filles, transcender la relation mère-fille. L'harmonie, c'est aussi la perception cosmogonique du réel qui sous-tend le récit, où la violence du monde se répercute dans

3 Cet essai peut aisément apparaître comme une mise en abyme de L'ange de la solitude. De fait, il sanctionne la jonction du discursif et du narratif: L'ange de la solitude, comme « De Sapho à Radclyffe Hall », entend décrypter les signes du monde. 
82

la vie de chacune. L'harmonie, c'est enfin l'équilibre de l'être, la résolution des valeurs contradictoires, l'apaisement de l'angoisse, le dosage de la sensualité et de l'ascétisme. Correspondance, analogie, synesthésie: peut-on chercher dans L'ange de la solitude la théorie fouriériste de l'harmonie, dont se réclame toute la poésie symboliste et surréaliste, de Rimbaud à Breton? Il me semble lire, dans cette doctrine esthétique, et ce sur tous les plans du texte, la motivation ultime de chacun des signes: de l'organisation sociale harmonienne à l'équilibre entre le corps, l'âme et l'intellect, jusqu'aux correspondances synesthésiques, l'utopie de Fourier se lit en filigrane. Cette pensée du continu, qui postule une relation triadique cosmos-logos-anthropos, investit toutes les pratiques signifiantes, même et surtout lorsqu'elles sont dysphoriques:

il y avait dans toute cette tapisserie en mouvement qui avait déplacé des sphères, en peu de temps, dans le monde de Johnie, une conjuration d'ordre divin, pensait Johnie, comme on en voit dans les astres qui reproduisent nos malheurs, de la naissance à la mort. Oui, ce devait être ainsi que s'exprimait la volonté de Dieu, pensait Johnie révoltée, et tout ce qui était d'ordre divin, comme la chute d'une étoile sur le toit terrestre, tranchait, rompait les vies. (AS, 36)

Mais l'utopie persiste: le réseau figuratif de la commune en est la concrétisation sur le plan narratif. Ce microcosme rappelle le phalanstère fouriériste, principe d'organisation sociale et politique où les humains vivent en communautés harmonieuses. Toute la problématique féministe exploitée par le texte s'y greffe aisément - les fouriéristes étaient partisans de l'émancipation de la femme - de même que le réquisitoire contre la guerre. Les personnages qui forment la commune traduisent le concept des correspondances verticales et horizontales du système analogique de Fourier: l'âme (Polydor), l'intellect (Johnie), le corps (Thérèse), alors que le champ synesthésique se voit repris par la peinture (L'Abeille), la musique (Doudouline), la drogue (Gérard). A cet égard, le travail synesthésique du texte, constant, vient préciser cette articulation fondamentale. Par exemple, on lit dans cet extrait à la fois la perception visuelle et tactile, et l'analogie du cosmos et de l'individuel:

car on ne pouvait plus avoir d'illusions après ce délire de couleurs dans les arbres et le ciel, ce serait la nuit, le froid 
dont on ne pouvait même pas peindre la couleur sur une toile, car c'était trop opaque, trop glacé, une matière si vile qu'elle causait des arrêts mortels du cœur, du flux sanguin, et Doudouline qui grattait sa guitare pendant que Polydor lui frôlait la joue de ses baisers. (AS, 96)

Même les correspondances entre les trois règnes, végétal, minéral, animal, seront tentées dans le texte: «elle était là comme une grande fleur dans sa chemise* $(A S, 23)$ ou *ces filles riant et chuchotant dans les poussières végétales que soulevaient les pneus de leurs bicyclettes dans la rue (AS, 36) ne relèvent pas de coïncidences fortuites. Il en est de même des personnages androgynes - et la figure est multipliée dans le texte - qui reprennent l'idée d'une harmonie homme-femme: les prénoms masculins des filles Gérard et Johnie en sont l'exemple privilégié, de même que Paula «qui est aussi le fils de sa mère» $(A S, 46)$.

Le seul couple euphorique du texte, Doudouline et Polydor, représente l'harmonie idéale, la fusion de la musique et du sacré, de l'âme et du corps, et rejoint l'analogie mystique mise en scène dans le texte par la lecture de saint Jean de la Croix:

même lorsqu'elles se voyaient tous les jours, elles s'écrivaient encore des poèmes, des chansons qu'elles se glissaient sous l'oreiller, le soir: ma chère Doudouline, mon cher amour, Polydor, mon petit saint Jean de la Croix. $(A S, 96)^{4}$

L'univers de Johnie, univers de signes à décrypter, univers harmonieux de correspondances signifiantes, d'analogies à la mesure de l'Univers, demeure utopique.

\section{"Le seuil de la douleur" ou l'inscription diffractée de signes analogiques}

*Le seuil de la douleur", titre de la seconde partie du texte, métaphorise la rupture, la perception du discontinu. Les signes épars du monde ne font plus sens. L'analogie

4 Les autres couples, aux amours difficiles, echouent. Ainsi, à propos de l'Abeille et de Thérése, le texte dira: a L'Abeille, oui, c'était là sans doute où la pensée de devenir deux avec une Thérèse métamorphosée en ordinateur, réservant pour l'Abeille tous les trésors de sa bonté et les merveilles de la technologie modeme, qu'elle parle ou qu'elle écrive, que le mariage avec l'âme rêvée s'était évanoui. " $(A S, 99)$ 
universelle, la correspondance des signes, la fusion harmonieuse, du côté du désir, se butent à la fragmentation, à la parcellisation, à la solitude. Dans cette perspective, la figure du plafond de la chambre de l'Abeille, figure récurrente du texte, vient préciser le rapport analogique que nous tentons de cerner:

lorsque tout n'était que stagnation sous les nuages de mars qui ne bougeaient pas, comme sous le plafond de la chambre dont l'Abeille avait compté toutes les rainures, chacune d'elles dissimulant un secret, un stigmate que le temps avait inscrit dans le plâtre, le bois, et il en était ainsi de chaque objet délaissé dans l'univers, la multiplicité des rainures finissait par vous vaincre, devenue le symbole de la répétition de nos gestes jusqu'à l'usure, la stérilité de l'ennui, le symbole aussi de notre abandon sur la terre, on s'endormait, pensait l'Abeille, lasse de ne rien comprendre au sens de sa vie... (AS, 18-19, c'est moi qui souligne).

À mon sens, le travail poétique du texte ressort de cette lecture symbolique du monde comme une réécriture signifiante de signes éclatés, partiels, voire contradictoires. Car le réseau textuel de la fragmentation, porté tant par le discours que par l'écriture, est omniprésent. Ce que j'appelle la fragmentation de la prose du monde s'inscrit dans le texte sous la forme de discours idéologiquement marqués, qui correspondent aux préoccupations contemporaines: l'écologie, le féminisme, le nouvel âge, l'ère des communications, la prostitution des jeunes, etc., s'inscrivent dans le texte en morceaux, comme des réquisitoires ponctuels contre tous les faits qui contreviennent à l'harmonie du monde. L'effet de réel indéniable engendré par ces références aux violences, aux famines et aux guerres masque mal la métaphorisation qu'elles autorisent. Ce discours * moralisateur *, lui-même fragmenté, vient s'ancrer aux autres lectures du monde que sont les voyages. Les lieux explorés apparaissent comme autant de parcelles du monde à décrypter: les pyramides aztèques, les prisons de Turquie, l'Éthiopie affamée, Jérusalem déchirée deviennent les «cratères de l'Histoire * où la souffrance du monde s'engloutit. En ce sens, le passage où Gérard se tient debout sur le discours du monde, inscrit un réseau métaphorique multiple, qui préfigure sa propre mort:

On avait déplié un journal sur la table où elle se tenait debout, il manquait encore une manche au costume, même sur ce 
papier du journal on voyait le feu et le sang qui se mêlaient et ces ombres squeiettiques sur les champs brûlés, au Soudan, le feu et le sang qui se mêlaient sous les yeux de la vieille dame qui cousait le bas du pantalon, pour sa fille, des batailles féroces se déroulaient, mais elle n'en savait rien, car ils iraient en Floride pour l'hiver, fuyant le froid et tout ce sang qui coulait ailleurs ou qui se coagulait comme pour le lapin assassiné, le lapin que Gérard avait reçu à Pâques avec les poussins dans la boîte, et plus tard, Gérard irait au Club dans son smoking dont le revers était en soie rouge. (AS, 79$)$

Le discours culturel est lui aussi fragmenté. Si l'on examine l'espace culturel ${ }^{5}$ mis en scène dans le texte, il appert que l'inscription récurrente de références à la musique, à la peinture et à la littérature participe du procès symbolique du texte. De fait, les trois pôles privilégiés par cette pratique citationnelle redoublent le narratif en ce sens qu'ils construisent des faisceaux de correspondances associés à chacun des personnages, faisceaux qui essaiment dans le texte entier. Ainsi, les références picturales à Gauguin ( $A S$, 39), Goya $(A S, 45)$, Van Gogh $(A S, 107)$ et Chagall $(A S, 105$, 107), appelées par les personnages de Paula et de l'Abeille présentés comme les peintres, se verront reprises respectivement par Doudouline pour dénigrer son frère, par L'Abeille pour caractériser une pose de Thérèse et par Johnie pour condamner Marianne qui fait le commerce des œuvres d'art. La fonction analogique de cette exploitation du corpus universel est ici évidente: les œuvres signalées renvoient, non pas seulement à une appréhension visuelle du monde, mais surtout à un jugement esthétique: *L'Art Nouveau * (AS, $31,47)$ est irrémédiablement condamné par Paula, qui peint en vitesse « une fresque sur la Grèce antique * $(A S, 31)$ pour son décor du lendemain. D'une part, le texte affiche ouvertement ses préférences envers les œuvres d'art qui incarnent la souffrance et il assigne, en ce sens, une fonction rédemptrice

5 Élaine Cliche, dans « Un rituel de l'avidité «, Voix \& images, vol. VIII, $n^{\circ} 2$, hiver 1983, p. 229-248, nomme espace culturel a le procès de nomination des lieux, des artistes et des ceuvres d'art " et lui assigne la fonction principale de décloisonner les textes. L'article identifie ces réseaux transtextuels dans Les nuits de l'underground (1978), Le sourd dans la ville (1979) et Visions d'Anna (1982), et les associe à un espace culturel ouvert opposé a la fermeture entropique du texte. Nous endossons cette lecture, dans la mesure où le champ culturel ouvert fonctionne à la façon d'un analogon de l'espace fermé, ou du discours fragmenté. 
86

à l'art; une telle position esthétique sera maintes fois réitérée. Les pratiques différentes de Paula et de l'Abeille l'une peint des arbres calcinés et l'autre des planètes multicolores pour exprimer sa douleur - viennent renforcer la polarisation entre deux démarches qui demeurent néanmoins liées dans leur volonté de symbolisation. D'autre part, le fait de réduire l'art pictural à la seule représentation n'est pas sans manifester un présupposé à l'effet que l'art doive d'abord et avant tout signifier. En ce sens, le tableau * Thérèse ou les plaisirs de l'été * 6 , comme une mélopée lancinante, insiste constamment sur le départ de Thérèse, qui n'a laissé de traces de son amour que ce portrait bucolique.

Les références musicales interviennent dans le texte pour ponctuer la distance entre les personnages. Michael Jackson $(A S, 17,22)$, Stevie Wonder (AS, 22), Ray Charles $(A S, 22)$, musique pop, vidéoclip, Madonna $(A S, 46)$, Tina Turner $(A S$, 104) s'opposent, comme il se doit, aux opéras wagnériens de Paula $(A S, 23,48)$, à la musique de Gounod $(A S, 29)$ et à la messe de Bach $(A S, 74)$. Les préférences musicales, constamment polarisées en musique populaire et musique classique, accentuent le caractère antithétique, volontiers manichéen de l'entreprise textuelle; la musique empêche la fusion des couples et signale le renversement des valeurs lié à la jeune génération. De ce point de vue, la transmission culturelle est toujours déviée, parcellisée: Cécile, la mère de l'Abeille, était une musicienne, fervente de Chopin, et un compositeur émérite (AS, 122), morte dans son sang. Sa fille sera l'amante d'une amie de sa mère, Paula. Sophie, la mère comédienne, a une fille musicienne. Seul l'écrit est en filiation directe: Johnie a hérité de ses parents, "féministes radicaux " dans le texte, la passion de la paperasse avec une insistance sur l'amour des livres, transmis par sa mère anglaise.

Le réseau figuratif littéraire se greffe autour du personnage de Sophie, la mère comédienne: Racine $(A S, 13)$, Strindberg ( $A S, 15,47,83)$, Molière $(A S, 81)$, Ibsen $(A S, 84)$, Claudel $(A S, 118)$ forment le répertoire joué par la mère à Paris. Les références philosophiques, Schopenhauer $(A S, 56)$, Marc-Aurèle $(A S, 44)$, Nietzsche et Rousseau $(A S, 117)$, sont liées aux personnages masculins. L'allusion au théâtre de

6 Ce tableau peint par l'Abeille deviendra, en seconde partie du texte, «Thérese ou les splendeurs de l'été". 
Michel Tremblay, "Il faudra que j'aille voir dans l'Est ", est vécue par Sophie comme une remise en question, cependant qu'ailleurs dans le texte on dénonce le snobisme qui nous fait préférer les écrivains étrangers. Le discours sur la littérature québécoise, bien que succinct, stigmatise, par une allusion à Hubert Aquin ${ }^{7}$, la dimension politique du littéraire qui empêche, semble-t-il, la reconnaissance de l'art:

mais pour l'Abeille, ce pays dont parlait Paula était un paradis libre et insensé où de grands poètes avaient été foudroyés par eux-mêmes, dans la cour des collèges, d'où le sang coulait encore, sur la neige, il était donc bien malfaisant, ce pays qui avait permis de telles ruptures dans les esprits, dans les consciences, ce pays dont l'incurable gloire des ainés, comme Paula, se nourrissait du sang, de la mort de ses artistes de génie? (AS, 70)

Ces ruptures semblent irrécupérables. La mémoire culturelle ne réussit pas à créer des liens harmonieux, pas plus que la religion, parcellisée en un panthéon de divinités. L'absence de discours unificateur équivaut à la perte de sens du monde. Ne demeurent que des fragments, douloureux, qui hantent la mémoire:

la disparition d'un être cher laissait en nous ces images inachevées, troublantes: longtemps nos âmes étaient lourdes de ces débris de choses, d'êtres vivants qui nous avaient quittés et dont le monde extérieur, soudain déformé, cauchemardeux, nous renvoyait l'image $(A S, 42)$

Toutes les figures thématisées du friable s'inscrivent de cette manière: à la dislocation des couples viennent se superposer toutes les figures de la cendre, de la cendre de cigarette qui ponctue la rupture entre Johnie et Linda à l'éparpillement des cendres de Gérard. Même la poudre blanche "sniffée » par Gérard est porteuse de mort: d'un transfert symbolique à l'autre, le système du texte sature toutes les entrées ${ }^{8}$. L'ange de la solitude, tout entier redevable de

7 Hubert Aquin revêt ici l'armure du poète maudit, au coeur de la poésie symboliste.

8 Les systèmes symboliques de l'ouvrage sont multiples. Par exemple, on aurait pu analyser avec profit cette scène, au symbolisme sexuel appuye, où un serpent tombe dans l'assiette de Paula (AS, 71) alors qu'elle est à expliquer à une Abeille inculte que Tlaloc est le dieu de la pluie et Quetzalcoatt le dieu de l'air et de l'eau. Dans cette perspective, tout ce passage où elles escaladent les 
88

l'analogie poétique, inscrit ses fragments épars, constamment repris, relus. Sans issue dès le départ, l'écriture échoue à donner un sens. Et comme pour renforcer encore cette lecture symboliste, même * les paradis artificiels * ne pourront contourner l'impasse. La dope, le hasch, qui élargissent dans le texte le champ perceptif synesthésique - et tout le jeu des sensations (massages, caresses, froid, chaleur), des odeurs, des couleurs, de la nourriture, de la voix - ne réussissent pas à recréer l'harmonie. Gérard, morte dans un incendie, ne laissera comme traces de son passage que ses souliers rouges et sa chaîne d'or avec une croix. La poésie symboliste ancre le narratif et en gère tous les signes.

\section{Aux frontières du poétique}

De la tension entre prose et poésie naît la spécificité de cette écriture. Le texte va tenter de résoudre, à tout le moins de concilier, dans son écriture même, deux pratiques textuelles codées, la romanesque et la poétique, en même temps qu'il les convoque dans le texte. On pourrait chercher, et trouver aisément, dans L'ange de la solitude, des marques formelles d'une écriture poétique. Ainsi, la structuration du texte en fragments textuels auto-engendrés ${ }^{9}$, qui oblige à une lecture tabulaire, et le jeu constant d'aplatissement temporel, où les souvenirs et réflexions s'entremêlent au temps de la narration, apparaîtraient comme des indices de neutralisation de l'écriture romanesque. En ce sens, les répétitions de séquences, de la plus large à la plus réduite, bouleversent l'ordre linéaire. Par exemple, le début de la seconde partie, où les personnages attendent Gérard, est quasi identique au début de la première, où les personnages attendent l'Abeille. Notons aussi la reprise in extenso du segment sur * la vision de leurs jambes retombant l'une sur l'autre, avec lassitude, dans le grand lit acheté à crédit » $(A S, 11,131)$. De telles séquences installent le texte dans une structure fermée, à

pyramides mayas n'est pas sans évoquer la poétique surréaliste et son engouement à l'égard des civilisations dites a primitives ". D'autre part, une étude bachelardienne, attentive aux éléments eau-air-terre-feu, révélerait sans doute d'autres correspondances, négligées ici.

9 Ainsi, le procédé mis en place dès les premières pages et qui se répétera constamment: du geste de Gérard dans une nuit silencleuse * pleine d'énigmes ", le texte enchaîne sur les prénoms a énigmatiques n des filles. 
temporalisation circulaire et sans dénouement. Mais l'exemple le plus probant serait encore de montrer l'accentuation rythmique du langage, telle qu'elle est manifestée dans l'extrait suivant:

Soudain, Johnie se séparait de Marianne, comme si elle eût été sa captive. De la cabine téléphonique où elle voyait le soleil couchant sur la mer, elle attendait, défaillante, la voix, le souffle de Lynda: la porte de la cabine était ouverte, Johnie respirait l'odeur entêtée de l'acacia, ce qui lui rappelait avec tristesse qu'elle avait trop bu de ce cocktail au rhum, la veille, avec Marianne. N'était-elle pas tombée dans un buisson d'acacias pour être recueillie ensuite contre la poitrine de Marianne qui avait ri, dans son costume blanc ? $(A S, 54)$

Cette séquence opère sur plusieurs plans: les reprises phoniques (allitération en $\mathrm{S}$ et $\mathrm{C}$, paronomase "buisson»/ "boisson") chevauchent les reprises syllabiques (captive, cabine, acacia), les répétitions lexicales (cabine et acacia) et les reprises sémantiques ("défaillante » renvoie à * tombée »). Il faut remarquer également le jeu synesthésique complet, de la vue à la voix, du souffle à l'odeur, de l'odeur au goût du cocktail, et du cocktail au contact avec Marianne qui, par son costume blanc, nous ramène à la vision de départ et à l'idée de séparation. Reprises, répétitions, structures en parallèle sont récurrentes, quasi constantes, dont le «je me noie, je me noie" de Paula, auquel fait écho le "ich komme, ich komme" de l'homme qui la sauve, ne serait que l'une des reprises syntaxiques les plus évidentes. Cependant, ce travail " poétique" du texte ne sert notre propos que dans la mesure où, s'imbriquant étroitement au discours romanesque, il accuse le double ancrage de L'ange de la solitude, tel qu'il se révèle par la pratique intertextuelle.

\section{De l'intertextualité comme procédé structurant}

Je ne retiendrai ici que les clins d'œil textuels les plus signifiants, soit l'exergue, tiré de Querelle de Brest de Jean Genet, et la strophe anonyme récitée à la toute fin du texte:

* Où t'es-tu caché, Ami

Toi qui me laisses dans les gémissements?

Pareil au cerf tu as fui, M'ayant navré après Toi, Je sortis criant et Tu étais parti. n (AS, 134) 
90

Cette strophe anonyme s'avère être la première du Cantique spirituel de saint Jean de la Croix ${ }^{10}$; nulle indication n'est mentionnée, seul le réseau monté autour de Polydor peut mettre sur la piste. À mon sens, c'est de la mise en perspective de ces deux indices diamétralement opposés que l'esthétique fondamentale de L'ange de la solitude se révélera. La distinction opérée ici aux fins d'analyse entre Genet et saint Jean de la Croix se motive aisément: l'exergue donne son titre à l'ouvrage alors que la strophe est la seule rupture dialoguée de tout le texte.

Plus que la dichotomie immédiate prose/poésie instaurée par ces références, plus encore que l'antithèse sacrilège/ mysticisme suggérée par le recours à Genet et saint Jean de la Croix, je retiens ici la tentative de résolution d'une esthétique duelle esquissée dans le texte. Posons d'abord la figure de la dualité, telle qu'elle est montrée dans le texte: à mon avis, la relation entre Johnie et Gérard traduit ce désir de fusion totale. Dans un premier temps, Johnie et Gérard métaphorisent, par les seules lettres de leurs prénoms, le JE du texte. L'indissociabilité de ces deux personnages est d'ailleurs inscrite dès le début du texte, dans ce passage ambigu où l'on ne distingue pas qui, de Johnie ou de Gérard, supporte l'autre:

* On gèle ici », avait soupiré Gérard, couchée là, sans frémissement sur l'ossature de Johnie, ses flancs durs, écoutant des chansons douceâtres à la radio. Gérard était paisible, comme évanouie, et Johnie avait senti sous elle cet assemblage d'os, de côtes maigres... $(A S, 12)$

D'autre part, le jeu anagrammatique accentue leur appartenance réciproque: Gérard évoque incontestablement Genet alors que Johnie n'est pas sans rappeler et Jean Genet et saint Jean de la Croix. Johnie survivra à Gérard. Si on lie à ce réseau les deux seuls objets calcinés qui demeurent de Gérard, soit * les souliers rouges et la chaînette d'or avec une croix à son extrémité " (AS, 123), la sanction de l'hypothèse posée est indéniable et le texte se voit immédiatement structuré par cette antinomie fondamentale. D'un côté, la technique romanesque est associée à Genet alors que le travail

10 Jean de la Croix, CEuvres completes, Paris, Desclee de Brouwer, 1967, p. 529-535. 
analogique du texte est renvoyé à saint Jean de la Croix. La ressemblance entre Querelle de Brest et L'ange de la solitude, hormis le thème de l'homosexualité mis en scène dans les deux textes, réside dans la multiplicité des figures qui leur sont communes. Le personnage de Jo Querelle, dans Querelle de Brest ${ }^{11}$, est l'Ange de la solitude $(Q B, 16)$; il a un frère, Robert, qui lui ressemble parfaitement, à tel point qu'on les confond:

Vous êtes seuls au monde, la nuit dans la solitude d'une esplanade immense. Votre double statue se réfléchit dans chacune de ses moitiés. Vous êtes solitaires et vivez dans votre double solitude. $(Q B, 158)$

Comme Johnie, Jo Querelle voyage de par le vaste monde; comme Gérard, il fait le trafic de la drogue. * La Féria *, le bordel de Querelle de Brest, ressemble étrangement au "Club " de L'ange de la solitude, de la même manière que Le vengeur, bateau de Querelle, évoque le Grand Mudder que Johnie regarde naviguer. $\AA$ ces indices anecdotiques, et ils sont multiples ${ }^{12}$, vient se greffer le travail de fragmentation du texte, qui offre des parentés évidentes, et une même association de l'homosexualité au rejet et à la mort (la figure nazie de l'Étoile d'Or ou de l'Étoile Rose est présente dans les deux textes). À ces considérations, il faut ajouter le travail synecdochique des deux textes. Querelle est toujours évoqué par "ses boucles noires", ses " souliers vernis noirs", son "pantalon bleu de fil", sa cravate et son slip de couleur "rouge", son «dos", son "béret» et surtout "ses pieds". «Beauté des pieds de Querelle» $(Q B, 83)$ dira le texte. Toutes ces figures seront reprises dans L'ange de la solitude et constamment réitérées: le smoking et les souliers de plastique rouges, le jean, les joues de marbre (qui préfigurent sa mort),

11 Je travaille avec la réédition de 1981 du texte de Jean Genet, Querelle de Brest, coll, "L'imaginaire ", Paris, Gallimard; les références renvoient à cette edition.

12 Une étude exhaustive des marques d'intertextualité s'avérerait fascinante: je n'ai retenu ici que les accents les plus évidents. Tout le réseau du friable semble s'y greffer: les tas de cendres, les tas de désespoir abondent autant dans les deux textes; il n'est jusqu'au meurtre du lapin qui ne rappelle Querelle de Brest, et la si belle phrase de Madame Lysiane: a Aurai-je donc la honte de me savoir Berthe aux grands pieds des sandales escaliers? Mais je suis davide " $(Q B, 161)$ qui rappelle à la fois les pieds particuliers du personnage Paula et le jeu textuel probable entre les pieds de tous les personnages des deux textes. 
sont les apanages de Gérard, son "armure de séduction *, alors que la "casquette* et le * dos $*$ métaphorisent les souffrances de Johnie. La beauté des pieds, ou leur faiblesse, figure omniprésente de L'ange de la solitude, se verra partagée par tous les personnages. Le rapport intertextuel multiplie les analogies.

À mon sens ce recours analogique au texte de Genet convoque - et c'est la spécificité de L'ange de la solitude l'écriture romanesque, la fragmente et la subordonne au poétique, dans une tentative de fusion du double. La figure de l'analogie, portée par toute la partie du texte qui renvoie à Querelle de Brest, se voit réinscrite sous celle de l'analogie poétique, portée par l'esthétique symboliste qui détermine L'ange de la solitude. Dans cette optique, la conclusion du texte par le poème "Cantiques entre l'âme et l'époux * de saint Jean de la Croix, semble boucler le réseau textuel: de l'analogie poétique à l'analogie mystique ${ }^{13}$, les arcanes sont franchies et l'Harmonie s'instaure. Tout le projet d'écriture de L'ange de la solitude se voit porté, comme par mimétisme, par cette lecture/écriture de l'analogie. Et la figure de Johnie, dans son rapport à l'écriture, endosse les attributs de sa mission: nimbée d'une aura particulière, elle seule, séparée de son double Gérard, peut décrypter les signes du monde pour accéder au sens.

\section{Une voix particulière}

L'ange de la solitude n'est pas un roman, dans la mesure où il en pervertit le code fondamental au profit d'une esthétique qui le détourne du narratif. Son architecture textuelle emprunte à la poésie symboliste et convoque, dans le texte, une myriade de discours fragmentés pour les installer en réseaux de correspondances statiques, jamais dynamisées.

13 André Breton, dans Signe ascendant, coll. « Poésie/Gallimard ", Paris, Gallimard, 1968, p. 9, pose en ces termes le rapport entre l'analogie poétique et l'analogie mystique: Considérée dans ses effets, il est vrai que l'analogie poétique semble, comme l'analogie mystique, militer en faveur de la conception d'un monde ramifié à perte de vue et tout entier parcouru de la méme séve mais elle se maintient sans aucune contrainte dans le cadre sensible, voire sensuel, sans marquer aucune propension à verser dans le surnaturel. Elle tend a faire entrevoir et valoir la vraie vie "absente" et, pas plus qu'elle ne puise dans la réverie métaphysique sa substance, elle ne songe un instant à faire tourner ses conquêtes à la gloire d'un quelconque "au-delà "." 
L'univers polyphonique romanesque n'existe plus: seul demeure un discours univoque, acharné à instaurer un sens, le sens. Louise Dupré dit de ce texte «qu'il demeure avant tout un livre où la quête d'amour se bute [...] sur la nécessité d'assumer une solitude qui ne soit ni exil ni errance, mais rencontre de sa propre voix "14. Et c'est bien de cela qu'il s'agit. Le lyrisme de L'ange de la solitude réside tout entier dans cette voix qui lie irrémédiablement, dans un transfert symbolique constant, la perte du sang à la perte du sens. Dans cette optique, l'écriture, de l'ordre du sacré, se doit d'endiguer cette violence; le rempart proposé sera celui de la reconquête de l'harmonie. L'analogie poétique, par le croisement dans l'espace et le temps d'univers éloignés, fictionnels et réels, culturels et politiques, donne à lire le sens du monde. Le roman contemporain nous avait pourtant détournés de cette entreprise démesurée. La voix particulière de MarieClaire Blais tente la réconciliation d'une poétique moderne tombée en désuétude et d'un formalisme romanesque postmoderne relativement essoufflé. Se refusant à l'une comme à l'autre, cette voix demeure résolument a-moderne.

14 Louise Dupré, « L'amour: cette autre identité ", Voix \& images, vol. $X V, n^{\circ} 2$, hiver 1990, p. 301. 